\title{
Many-valued Intuitionistic Implication and Inference Closure in a Bilattice-based Logic
}

\author{
Zoran Majkić \\ Dept. of Computer Science,UMIACS, University of Maryland, College Park, MD 20742 \\ zoran@cs.umd.edu \\ http://www.cs.umd.edu/ zoran/
}

\begin{abstract}
In this paper we present a many-valued logic programming, based on reinterpreted Belnap's 4-valued bilattice: we introduce the new semantics for a 4-valued implication by relative pseudo-complement, used for intuitionistic logics. This kind of logic programming is particularly useful for data integration with possibly incomplete and inconsistent information. We define an ontological encapsulation of the epistemic many-valued logic programs with negation, based on this bilattice, into 2valued meta logic programs. Obtained 2-valued logic semantically reflects original epistemic many-valued logic, and can be used in order to define many-valued logic entailment and inference closure for many-valued truth assignments.
\end{abstract}

\section{INTRODUCTION}

Generally, three-valued, or partial model semantics has had an extensive development for logic programs, [1], [2]. Przymusinski extended the notion of the stable model to allow 3-valued, or partial, stable models, [3], and showed that every program has at least one partial stable model, and that the wellfounded model is the smallest among them, [4]. Once one has made the transition from classical to partial models allowing incomplete information, it is a small step towards allowing models admitting inconsistent information: the idea is that we may define the semantics of logic programs with inconsistent information by the smallest partial stable 4-valued models. Doing so provides a natural framework for the semantic understanding of logic programs that are distributed over several sites, with possible conflicting information coming from different places.

By a logic program we mean a finite set of universally quantified clauses of the form $\forall\left(A \leftarrow L_{1} \wedge \ldots \wedge L_{m}\right)$, and a set of constraints $\forall\left(\leftarrow L_{1} \wedge \ldots \wedge L_{m}\right)$, where $m \geq 0$, $A$ is an atom and $L_{i}$ are positive or negative literals (see [5]). Following a standard convention, such clauses will be simply written as clauses of the form $A \leftarrow L_{1} \wedge \ldots \wedge L_{m}$.

The alphabet of a program $P$ consists of all constants, predicate and functional symbols that appear in $P$, a countably infinite set of variable symbols, connectives $(\wedge, \vee, \sim, \leftarrow$ i.e., and, or, not and logic implication, respectively), and the usual punctuation symbols. We assume that if $P$ does not contain any constant, then one is added to the alphabet. The language $\mathcal{L}$ of $P$ consists of all the well-formed formulae of the so obtained first order theory. We assume that the Herbrand universe is $\Gamma_{U}=\Gamma \bigcup \Omega$, where $\Gamma$ is ordinary domain of database constants, and $\Omega$ is an infinite enumerable set of marked null values, $\Omega=\left\{\omega_{0}, \omega_{1}, \ldots.\right\}$, and for a given logic program $P$ composed of a set of predicate and function symbols, $P_{S}, F_{S}$ respectively, we define a set of all terms, $\mathcal{T}_{S}$, and its subset of ground terms $\mathcal{T}_{0}$, then atoms are defined as: $\mathcal{A}_{S}=\left\{p\left(c_{1}, . ., c_{n}\right) \mid p \in P_{S}, n=\operatorname{arity}(p)\right.$ and $\left.c_{i} \in \mathcal{T}_{S}\right\}$ The Herbrand base, $H_{P}$, is the set of all ground (i.e., variable free) atoms.

In [6], Belnap introduced a logic intended to deal in a useful way with inconsistent or incomplete information. It is the simplest example of a non-trivial bilattice and it illustrates many of the basic ideas concerning them. We denote the four values as $\mathcal{B}=\{t, f, \mathrm{~T}, \perp\}$, where $t$ is true, $f$ is false, $\mathrm{T}$ is inconsistent (both true and false) or possible, and $\perp$ is unknown.

As Belnap observed, these values can be given two natural orders: truth order, $\leq_{t}$, and knowledge order, $\leq_{k}$, such that $f \leq_{t} \mathrm{~T} \leq_{t} t, \quad f \leq_{t} \perp \leq_{t} t$, and $\perp \leq_{k} f \leq_{k} \mathrm{~T}, \quad \perp \leq_{k} t \leq_{k} \mathrm{~T}$. This two orderings define corresponding equivalences $=_{t}$ and $={ }_{k}$. Thus any two members $\alpha, \beta$ in a bilattice are equal, $\alpha=\beta$, if and only if (shortly 'iff' ) $\alpha={ }_{t} \beta$ and $\alpha={ }_{k} \beta$.

Meet and join operators under $\leq_{t}$ are denoted $\wedge$ and $\vee$; they are natural generalizations of the usual conjunction and disjunction notions. Meet and join under $\leq_{k}$ are denoted $\otimes$ (consensus, because it produces the most information that two truth values can agree on) and $\oplus$ (gullibility, it accepts anything it's told), such that hold:

$f \otimes t=\perp, f \oplus t=\mathrm{T}, \mathrm{T} \wedge \perp=f$ and $\mathrm{T} \vee \perp=t$.

There is a natural notion of truth negation, denoted $\sim$, (reverses the $\leq_{t}$ ordering, while preserving the $\leq_{k}$ ordering): switching $f$ and $t$, leaving $\perp$ and $\mathrm{T}$, and corresponding knowledge negation, denoted - , (reverses the $\leq_{k}$ ordering, while preserving the $\leq_{t}$ ordering):switching $\perp$ and $\mathrm{T}$, leaving $f$ and $t$. These two kind of negation commute: $-\sim x=\sim-x$ for every member $x$ of a bilattice.

It turns out that the operations $\wedge, \vee$ and $\sim$, restricted to $\mathcal{B}_{3}^{\perp}=\{f, t, \perp\}$ are exactly those of Kleene's strong 3-valued logic. A more general information about bilattice may be found in [7]. The Belnap's 4-valued bilattice is infinitary distributive. In the rest of this paper we denote by $\mathcal{B}_{4}$ ( or simply $\mathcal{B}$ ) this 4 -valued Belnap's bilattice, and by $\mathcal{B}_{3}^{\perp}, \mathcal{B}_{3}^{\top}$ its 3-valued sublattices (the first is used for a 3-valued Kleene's logic (programs with incomplete, unknown information), the second for complete but inconsistent information).

As we can see, this bilattice logic does not contain the definition of the many-valued logic implication operator. In this paper we will enrich this billatice with the implication 
operator based on the intuitionistic semantics.

A (ordinary) Herbrand interpretation is a many-valued mapping $I: H_{P} \rightarrow \mathcal{B}$. If $P$ is a many-valued logic program with the Herbrand base $H_{P}$, then the ordering relations and operations in a bilattice $\mathcal{B}_{4}$ are propagated to the function space $\mathcal{B}_{4}^{H_{P}}$, that is the set of all Herbrand interpretations (functions), $I=v_{B}: H_{P} \rightarrow \mathcal{B}_{4}$.

Definition 1 Ordering relations are defined on the Function space $\mathcal{B}_{4}^{H_{P}}$ pointwise, as follows: for any two Herbrand interpretations $v_{B}, w_{B} \in \mathcal{B}_{4}^{H_{P}}$

1. $v_{B} \leq_{t} w_{B}$ if $v_{B}(A) \leq_{t} w_{B}(A)$ for all $A \in H_{P}$.

2. $v_{B} \leq_{k} w_{B}$ if $v_{B}(A) \leq_{k} w_{B}(A)$ for all $A \in H_{P}$.

3. $\sim v_{B}$, such that $\left(\sim v_{B}\right)(A)=\sim\left(v_{B}(A)\right)$.

4. $-v_{B}$, such that $\left(-v_{B}\right)(A)=-\left(v_{B}(A)\right)$.

It is straightforward [8] that this makes a function space $\mathcal{B}_{4}^{H_{P}}$ itself a complete infinitary distributive bilattice. Ginsberg [9] defined a world-based bilattices, considering a collection of worlds $W$, where by world we mean some possible way of how things might be:

Definition 2 [9] A pair $[U, V] \in \mathcal{P}(W) \times \mathcal{P}(W)$ of subsets of $W$ (here $\mathcal{P}(W)$ denotes the powerset of the set $W$ ) express truth of some sentence $p$, with $\leq_{t}, \leq_{k}$ truth and knowledge preorders relatively, as follows:

1. $U$ is a set of worlds where $p$ is true, $V$ is a set of worlds where $p$ is false, $P=U \cap V$ is a set where $p$ is inconsistent (both true and false), and $W-(U \cup V)$ where $p$ is unknown.

2. $[U, V] \leq_{t}\left[U_{1}, V_{1}\right] \quad$ iff $U \subseteq U_{1}$ and $V_{1} \subseteq V$

3. $[U, V] \leq_{k}\left[U_{1}, V_{1}\right] \quad$ iff $U \subseteq U_{1}$ and $V \subseteq V_{1}$.

The bilattice operations associated with $\leq_{t}$ and $\leq_{k}$ are:

4. $[U, V] \wedge\left[U_{1}, V_{1}\right]=\left[U \cap U_{1}, V \cup V_{1}\right]$,

$[U, V] \vee\left[U_{1}, V_{1}\right]=\left[U \cup U_{1}, V \cap V_{1}\right]$

5. $[U, V] \bullet\left[U_{1}, V_{1}\right]=\left[U \cap U_{1}, V \cap V_{1}\right]$,

$[U, V]+\left[U_{1}, V_{1}\right]=\left[U \bigcup U_{1}, V \bigcup V_{1}\right]$

6. $\sim[U, V]=[V, U]$.

Let denote by $\mathcal{B}_{W}$ the set $\mathcal{P}(W) \times \mathcal{P}(W)$, then the structure $\left(\mathcal{B}_{W}, \wedge, \vee, \bullet,+, \sim\right)$ is a bilattice.

Such definition is well suited for 3-valued Kleene logic, but not for 4-valued logic: for example, let $W=[1,100]$ be the closed interval of integers (indexes for a collection of worlds), $U=[1,60], V=[50,100]$, and $U_{1}=[1,60], V_{1}=[40,100]$; then $[U, V] \leq_{t}\left[U_{1}, V_{1}\right]$ while $T=U-P=[1,50] \supset[1,40]=$ $U_{1}-P_{1}=T_{1}$, and $F=F_{1}=[60,100]$ ! The other reason is that in this case we can't assign $T$ to any sentence, otherwise we will obtain an inconsistent theory where all sentences are inconsistent.

Consequently, we adopt a triple $[T, P, F]$ of mutually disjoint subsets of $W$ to express truth of some sentence $p$ (the $W-T \bigcup P \bigcup F$ is a set of worlds where $p$ is unknown), with the following definition for their truth and knowledge orders: $2.1[T, P, F] \leq_{t}\left[T_{1}, P_{1}, F_{1}\right]$ iff $T \subseteq T_{1}$ and $F_{1} \subseteq F$ $2.2[T, P, F] \leq_{k}\left[T_{1}, P_{1}, F_{1}\right]$ iff $T \subseteq T_{1}, P \subseteq P_{1}$ and $F \subseteq F_{1}$.

The meet and join truth and knowledge operations for this extended bilattice can be found in [10]. In this way we consider the possible value as weak true value and not as inconsistent (that is both true and false), and, consequently, we do not consider them to establish truth ordering (our Def
2.1 differs from Ginsberg's Def.). We have more knowledge for ground atom with such value, w.r.t. the true ground atom, because we know also that if we assign the true value to such atom we may obtain an inconsistent database.

The difference of a possible and unknown value may be explained also intuitively as follows: if we consider a 3-valued Kleene's strong logic, and try to use it in order to give a semantics for databases with inconsistencies, then we will obtain a number of stable 3-valued models (minimal 'repairs') for it. In each such stable model the set of unknown ground atoms is invariant: if one atom is unknown in some model it remains unknown in all other stable models. But we will have some atom true in some and false in some other stable model: to such atoms we can assign the possible logic value in a framework of this 4-valued logic, in order to obtain a unique minimal Herbrand model. Because of that, I prefer the Lukasiewicz's term "possible" for bilattice top-knowledge logic value T, and Kleene's term "unknown" for bilattice bottom-knowledge logic value $\perp$.

In [11] is given the definition for modal operators on bilattices, which generalizes both Kripke's possible world approach and Moore's autoepistemic logic: a modal operator is any n-ary function from the bilattice $\mathcal{B}$ to itself, with the following property:

Definition 3 [11] A modal operator on a bilattice $\mathcal{B}$ will be called deductive if and only if it commute with $\otimes$ and $\oplus$. All other modal operators will be called nondeductive.

For example, a nondeductive modal operator is Moore's operator $M$, [12], where $M p$ is intended to capture the notion of, "I know that p", that is, $M p=t$, if value of $p$ is $t$ or $\mathrm{T}$; $f$ otherwise.

This operator we introduced for the Autoepistemic manyvalued Logic Programming [13], in order to relax a belief on ground facts and be able to reason in the presence of the inconsistent information also: a ground fact, $p \leftarrow t$, is substituted by a modal formula $M(p \leftarrow t)$. The head of each integrity constrain for such programs that admit inconsistent information is changed from $f$ to $T$ logic value.

The plan of this paper is the following: Section 2 defines the 4-valued bilattice inference requirements and is defined many-valued intuitionistic implication for rules of a 4-valued logic programming. Such formal definition for many-valued implication is used in Section 3, where is shortly presented model theoretic Herbrand semantics, [14], [15] for the ontologically encapsulated many-valued (EMV) logic programmes (transformation into a 2-valued logic programs). The semanticreflection of the epistemic into the ontological framework, defines the semantic of this operator $\mathcal{E}$. Such ontological encapsulation is a necessary tool in order to define the many-valued logic inference, and is used also for coalgebraic semantics (equal to the well-founded semantics) of general logic programs [16].

In Section 4 we discuss the world-based closure for the epistemic truth assignment based on the deduction closure of the 2-valued logic at the ontological level. Finally, in Section 5 is given the answer to the, by Ginsberg and Schaerf, open problem: we present the inference closure for the truth assignments in many-valued logics. 


\section{MANY-VALUED INTUITIONISTIC IMPLICATION}

One of the key insights behind bilattices [9], [7] was the interplay between the truth values assigned to sentences and the (non classic) notion of implication. The problem was to study how truth values should be propagated "across" implications. The constructivism is surprisingly close to logic programming [17]. The features of logic programming that are unconventional from the classical point of view find immediate constructivistic explanations. Constructivism does not allow indefiniteness in proofs: from a constructivistic viewpoint implications are not "hidden disjunctions". Constructivism is causalistic: implications are viewed as inferring new information from already proved information, like in logic programming.

In this paragraph we will try to introduce the formal definition of many-valued implication for logic programs. Notice that logic implication and logic entailment, as pointed by Belnap, for the 4-valued logic are strongly connected: the implication has to be the principal structure for inference (entailment) capabilities. Let denote by $\vDash_{B}$ this bilattice 4-valued entailment, this this paradigm can be defined as follows: ${ }^{\prime} p \vDash_{B} q$ iff $p \rightarrow q$ is true".

From this point of view, we are fundamentally interested only for the cases when an implication is true. That is, at least from my point of view, the reason why in many-valued logic programming (e.g., Fitting, Przymusinski 3-valued logic) the implication is, differently from other logic connectives, defined as two-valued connective, such that it preserves truth: $p \rightarrow q$ just in case for each assignment of one of four values to the variables, the truth value of $p$ does not exceed the value of $q$ (in symbols: $v(p) \leq_{t} v(q)$ for each truth assignment $v: \mathcal{L} \rightarrow \mathcal{B}$ ). In order to obtain such many-valued definition, which generalizes the 2-valued definition given above we will consider the conservative extensions of Lukasiewicz's and Kleene's strong 3 -valued matrices (where third logic value $\perp$ is considered as unknown) in the intuitionistic way. Such conservative extensions are based on the observation we explained in precedence: the problem to study how the truth values should be propagated "across" implications can be restricted only to true implications (in fact we don't use implications when they are not true, because the 'immediate consequence operator' derives new facts only for true clauses, i.e. when implication is true). Thus, what we must guarantee is that if $b \leftarrow a$ and $c \leftarrow b$ are true then $c \leftarrow a$ also must be true, in order to guarantee the reflexivity and the transitivity of the logic entailment $\vDash_{B}$. In other words, that law is intimately connected with inference fixpoint semantics for logic programs: let us consider that in the i-th step the ground clause $b \leftarrow a$ become true, so that we derive the new fact $b$ from body $a$, and that in some $i+k$-th step the ground clause $c \leftarrow b$ becomes true, so that we derive the new fact $c$ : it means that $c \leftarrow a$ has to be true.

With such constructivistic considerations, Heyting produced an axiomatic system of propositional logic which claimed to generate as theorems precisely those sentences that are valid according to the intuitionistic conception of truth. It is well known that the implication for intuitionistic logic satisfies the conditions above. It is defined by the relative pseudo- complement [18] as follows:

the logical value of intuitionistic implication $a \rightarrow b$ is the greatest member $c$ of $\mathcal{B}$ (w.r.t. the truth ordering) such that $a \wedge c \leq_{t} b$ (that is $a \rightarrow b \equiv \bigvee\left\{c \mid a \wedge c \leq_{t} b\right\}$ ). Thus we obtain that $a \rightarrow b$ iff $a \leq_{t} b$ (for $c=t$ ), and that the modus ponens inference rule holds: if $a \rightarrow b$, that is, $a \leq_{t} b$, and $a$ is true, then $t \leq_{t} b$, that is, $b$ must be true.

The relative pseudo-complement for finite distributive lattices always exist. In fact, the intuitionistic implications, $\rightarrow \frac{1}{3}, \rightarrow_{3}^{\mathrm{T}}$, for two sublattices $\mathcal{B}_{3}^{\perp}, \mathcal{B}_{3}^{\top}$, respectively, are uniquely defined by the following truth-functional tables

\begin{tabular}{|c|c|c|c|}
\hline$\rightarrow \frac{1}{3}$ & $t \perp f$ & $\rightarrow_{3}^{\top}$ & $t \quad \mathrm{~T} f$ \\
\hline$t$ & $t \perp f f$ & $t$ & $t \quad \mathrm{~T} f$ \\
\hline$\perp$ & $t \quad t \quad f$ & T & $t \quad t \quad f$ \\
\hline$f$ & $t \quad t \quad t$ & $f$ & $t \quad t \quad t$ \\
\hline
\end{tabular}

The unique conservative intuitionistic extension of these two 3 -valued implications into the 4-valued implication is given by the following truth-matrix, $f_{\leftarrow}: \mathcal{B} \times \mathcal{B} \rightarrow \mathcal{B}$

(we denote by _* and _+ the modified and the new defined values w.r.t. the 3 -valued cases above, respectively):

\begin{tabular}{|c|cccc|}
\hline$\rightarrow$ & $t$ & $\perp$ & $f$ & $\mathrm{~T}$ \\
\hline$t$ & $t$ & $\perp$ & $f$ & $\mathrm{~T}$ \\
$\perp$ & $t$ & $t$ & $\mathrm{~T}^{*}$ & $\mathrm{~T}^{+}$ \\
$f$ & $t$ & $t$ & $t$ & $t$ \\
$\mathrm{~T}$ & $t$ & $\perp^{+}$ & $\perp^{*}$ & $t$ \\
\hline
\end{tabular}

Remark: The negation in the intuitionistic logic is defined by the pseudo-complement, that is, $\neg \alpha$ is equivalent to $\alpha \rightarrow f$, i.e., $\neg \alpha$ is the l.u.b. of $\{\beta \mid \alpha \wedge \beta=f\}$, so that " $\neg$ " is different from the epistemic negation " $\sim$ " (it is also different from the knowledge bilattice negation " - ") and, consequently, we do not need it for the logic programming.

For our purpose we obtained the Lukasiewicz's extension and a tautology $a \leftarrow a$ for any formula $a$. From intuitionistic semantics for this implication we guarantee the truth of a clause (implication) $p(\mathbf{c}) \leftarrow B$, whenever (iff) $v_{B}(p(\mathbf{c})) \geq_{t} v_{B}(B)$ , as used in fixpoint semantics for 'immediate consequence operators'. It is easy to verify that the 3-valued intuitionistic implications are sceptical reductions of $\rightarrow$ : the values in excess are replaced by $f$ (false).

We define also the restriction to 2 -valued logic implication $\leftarrow_{2}$, such that its matrix, $f_{\leftarrow 2}: \mathcal{B} \times \mathcal{B} \rightarrow \mathbf{2}$, where $\mathbf{2}=\{t, f\}$, is defined by: for any $\alpha, \beta \in \mathcal{B}, \quad f_{\leftarrow_{2}}(\alpha, \beta)=t$ iff $f_{\leftarrow}(\alpha, \beta)=t ; \quad f$ otherwise.

Thus, the valuations can be extended to maps from the set of all ground (variable free) formulas to $\mathcal{B}$ in the following way:

Definition 4 Let $\mathcal{P}_{S}$ be the set of all predicate symbols $\left(\mathcal{P}_{B} \subseteq\right.$ $\mathcal{P}_{S}$ is a subset of built-in predicates), $\boldsymbol{e}$ the special (error) constant, and $I: H_{P} \rightarrow \mathcal{B}$ be a many-valued Herbrand interpretation. A valuation I determines:

1. A Generalized interpretation mapping $\mathcal{I}: \mathcal{P}_{S} \times \bigcup_{i \leq \omega} \mathcal{T}_{0}^{i} \rightarrow$ $\mathcal{B} \bigcup\{\boldsymbol{e}\}$, such that for any $\boldsymbol{c}=\left(c_{1}, . ., c_{n}\right) \in \mathcal{T}_{0}^{n}, \quad \overline{\mathcal{I}}(p, \boldsymbol{c})=$ $I(p(\boldsymbol{c}))$ iff $\operatorname{arity}(p)=n ; \boldsymbol{e}$ otherwise.

2. A unique valuation map, also denoted $v_{\mathcal{B}}: \mathcal{L} \rightarrow \mathcal{B}$, on the set of all ground formulas $\mathcal{L}$, according to the following conditions: $\quad v_{\mathcal{B}}(\sim X)=\sim v_{\mathcal{B}}(X)$ and 
$v_{\mathcal{B}}(X \odot Y)=v_{\mathcal{B}}(X) \odot v_{\mathcal{B}}(Y)$, where $\odot \in\{\wedge, \vee, \otimes, \oplus \leftarrow\}$ 3. A truth assignment $u_{B}: \mathcal{L} \rightarrow \mathcal{B}$ will be called an extension of a truth assignment $v_{B}$ if $u_{B}(\psi) \geq_{k} v_{B}(\psi)$ for all $\psi \in \mathcal{L}$. If $u_{B}$ is an extension of $v_{B}$, we will write $u_{B} \geq_{k} v_{B}$.

\section{MANY-VALUED ONTOLOGICAL ENCAPSULATION}

The many-valued logic is based on four bilattice values which are epistemic. Sentences are to be marked with some of these bilattice logic values, according as to what the computer has been told; or, with only a slight metaphor, according to what it believes or knows.

Of course these sentences have also Frege's ontological truthvalues (true and false), independently of what the computer has been told: we want that the computer can use also these ontological 'meta' knowledge. Let, for example, the computer believes that the sentence $p$ has a value T (possible); then the 'meta' sentence,'I (computer) believe that $p$ has a possible value" is ontologically true. The many-valued encapsulation, defined as follows, is just the way to pass from the epistemic ('object') many-valued logic into ontological ('meta') 2-valued logic.

Such encapsulation is characterized by having capability for semantic-reflection: intuitively, for each predicate symbol we need some function which reflects its logic semantic over a domain.

Let us introduce also the set of functional symbols $\kappa_{p}$ in our logical language in order to obtain an enriched logical language where we can encapsulate the many-valued logic programming. Such set of functional symbols will be derived from the following Bilattice-semantic mapping $\mathcal{K}$ :

Definition 5 A semantic-reflection is a mapping $\mathcal{K}: \mathcal{P}_{S} \rightarrow$ $(\mathcal{B} \bigcup\{\boldsymbol{e}\})^{\bigcup_{i \leq \omega} \mathcal{T}_{0}^{i}}$, and we denote shortly $\kappa_{p}=\mathcal{K}(p)$ : $\bigcup_{i \leq \omega} \mathcal{T}_{0}^{i} \rightarrow \mathcal{B} \bigcup\{\boldsymbol{e}\}, \quad p \in \mathcal{P}_{S}$, such that for any $\boldsymbol{c}=$ $\left(c_{1}, . ., c_{n}\right) \in \mathcal{T}_{0}^{n}$, holds: $\quad \kappa_{p}(\boldsymbol{c})=\boldsymbol{e} \quad$ iff $\quad \operatorname{arity}(p) \neq n$.

If $p$ is a built-in predicate, then a mapping $\kappa_{p}$ is uniquely defined by: for any $\boldsymbol{c} \in \mathcal{T}_{0}^{n}, n=\operatorname{arity}(p)$, holds that $\kappa_{p}(\boldsymbol{c})=t$ if $p(\boldsymbol{c})$ is true; $f$ otherwise.

The many-valued ground atoms of a bilattice-based logical language $\mathcal{L}_{\mathcal{B}}$ can be transformed in 'encapsulated' atoms of a 2-valued logic in the following simple way: the original (many-valued) fact that the ground atom $p\left(c_{1}, . ., c_{n}\right)$, of the n-ary predicate $p$, has an epistemic value $\alpha=\kappa_{p}\left(c_{1}, . ., c_{n}\right)$ in $\mathcal{B}_{4}$, we transform in the new ground atom $p^{A}\left(x_{1}, \ldots, x_{n}, \alpha\right)$ with meaning "it is true that $p\left(c_{1}, . ., c_{n}\right)$ has a value $\alpha$ ". That is, we replace the original $\mathrm{n}$-ary predicate $p\left(x_{1}, . ., x_{n}\right)$ with $\mathrm{n}+1$-ary predicate $p^{A}\left(x_{1}, . ., x_{n}, \alpha\right)$, with the added logicattribute $\alpha$. It is easy to verify that for any given manyvalued valuation $v_{\mathcal{B}}$, every ground atom $p^{A}\left(c_{1}, . ., c_{n}, \alpha\right)$ is ontologically true (when $\alpha=v_{B}\left(p\left(c_{1}, . ., c_{n}\right)\right)$ ) or false. Let EMV denote this new 2-valued encapsulation of many-valued logic.

We distinguish between what the reasoner believes in (at the object (epistemic many-valued sublanguage) level), and what is actually true or false in the real world (at the EMV ontological 'meta' level), thus, roughly, the 'meta' level is an (classic) encapsulation of the object level.
Definition 6 Let $P$ be an 'object' many-valued logic program with the set of predicate symbols $P_{S}$. The translation in the encapsulated syntax version in $P^{A}$ is as follows:

1. For any $\alpha \in \mathcal{B}$, considered as 0 -ary predicate (bilattice constant), $\quad \mathcal{E}(\alpha)=\alpha^{A}(\alpha)$, where $\alpha^{A}\left({ }_{-}\right)$is an unary bilattice predicate over domain of bilatice values, such that for any $\alpha, \beta \in \mathcal{B}, \alpha^{A}(\beta)=t$ if $\alpha=\beta ; \quad f$ otherwise;

2. Each positive literal in $P$, $\mathcal{E}\left(p\left(x_{1}, . ., x_{n}\right)\right)=p^{A}\left(x_{1}, . ., x_{n}, \kappa_{p}\left(x_{1}, . ., x_{n}\right)\right)$;

3. Each negative literal in $P$,

$\mathcal{E}\left(\sim p\left(x_{1}, . ., x_{n}\right)\right)=p^{A}\left(x_{1}, . ., x_{n}, \sim \kappa_{p}\left(x_{1}, . ., x_{n}\right)\right) ;$

4. $\mathcal{E}(\phi \wedge \varphi)=\mathcal{E}(\phi) \wedge \mathcal{E}(\varphi) ; \quad \mathcal{E}(\phi \vee \varphi)=\mathcal{E}(\phi) \vee \mathcal{E}(\varphi) ;$

5. $\mathcal{E}(\phi \leftarrow \varphi)=\mathcal{E}(\phi) \leftarrow^{A} \mathcal{E}(\varphi)$, where $\leftarrow^{A}$ is a new syntax symbol for the implication at the encapsulated 2-valued 'meta' level;

6. For any modal formula on ground facts,

$\mathcal{E}\left(M\left(p\left(c_{1}, \ldots, c_{n}\right) \leftarrow t\right)\right)=p^{A}\left(c_{1}, \ldots, c_{n}, t\right) \vee p^{A}\left(c_{1}, \ldots, c_{n}, \mathrm{~T}\right)$.

Thus, the obtained 'meta' program is $P^{A}=\{\mathcal{E}(\phi) \mid \phi$ is a clause in $P\}$, with the 2-valued Herbrand base $H_{P}^{A}=$ $\left\{p^{A}\left(c_{1}, . ., c_{n}, \alpha\right) \mid p\left(c_{1}, . ., c_{n}\right) \in H_{P}\right.$ and $\left.\alpha \in \mathcal{B}\right\}$

This embedding of the many-valued logic program $P$ into a 2-valued 'meta' logic program $P^{A}$ is an ontological embedding: it views formulae of $P$ as beliefs and interprets negation $\sim p\left(x_{1}, . ., x_{n}\right)$ in rather restricted sense - as belief in the falsehood of $p\left(x_{1}, . ., x_{n}\right)$, rather as not believing that $p\left(x_{1}, . ., x_{n}\right)$ is true (like in an ontological embedding for classical negation).

Like for Moore's autoepistemic operator, for the encapsulation operator $\mathcal{E}, \mathcal{E} \phi$ is intended to capture the notion of, "I know that $\phi$ has a value $v_{B}(\phi)$ ", for a given valuation $v_{B}$ of the many-valued logic program.

Let $\mathcal{L}$ be the set of all ground well-formed formulae defined by this Herbrand base $H_{P}$ and bilattice operations (included many-valued implication $\leftarrow$ also), with $\mathcal{B} \subseteq \mathcal{L}$. We define the set of all well-formed encapsulated formulae by:

$\mathcal{L}^{A}=_{\text {def }}\{\mathcal{E}(\psi) \mid \psi \in \mathcal{L}\}$, so that $H_{P}^{A} \subseteq \mathcal{L}^{A}$, thus we obtain

Proposition 1 The encapsulation operator $\mathcal{E}$ is :

1. Homomorphism between the 'object' algebra $(\mathcal{L}, \wedge, \vee, \leftarrow)$ with carrier set of (positive and negative) literals, and 'meta' algebra $\left(\mathcal{L}^{A}, \wedge^{A}, \vee^{A}, \leftarrow^{A}\right)$, where $\wedge^{A}, \vee^{A}$ are 2-valued reductions of bilattice meet and join, respectively, denoted by $\wedge, \vee$ also.

2. The 'meta' implication operator $\leftarrow^{A}$ is not 2-valued operator: its matrix is given by $f_{\leftarrow A}: \mathcal{B} \times \mathcal{B} \rightarrow 2$, where $f_{\leftarrow A}=\mathcal{E} \circ f_{\leftarrow}=f_{\leftarrow 2}$.

Proof: The points 1 and 2 are direct consequences of the Definition 6. The matrix of 'meta' implication is equal to the matrix of $\leftarrow_{2}$ (2-valued restriction of $\leftarrow$ ) analogously to 2-valued implication used in Fitting's fixpoint semantics of immediate consequence operator for logic programs. Moreover, the semantics of 'meta' implication is a direct consequence of modal operator $\mathcal{E}$ and many-valued intuitionistic implication $\leftarrow$.

Notice, that with the transformation of the original 'object' logic program $P$ into its ontological 'meta' version program 
$P^{A}$ we obtain positive consistent logic program.

A Herbrand interpretation of $P^{A}$ is a 2-valued mapping (ainterpretation) $I^{A}: H_{P}^{A} \rightarrow \mathbf{2}$. We denote by $\mathbf{2}^{H_{P}^{A}}$ the set of all interpretations (functions) from $H_{P}^{A}$ into $\mathbf{2}$, and by $\mathcal{B}^{H_{P}}$ the set of all consistent Herbrand many-valued interpretations, from $H_{P}$ to the bilattice $\mathcal{B}$.

The meaning of the encapsulation of this many-valued logic program $P$ into this 'meta' logic program $P^{A}$ is fixed into the kind of interpretation to give to such new introduced functional symbols $\kappa_{p}=\mathcal{K}(p)$ : in fact we want [14] that they reflect (encapsulate) the semantics of the 'object' level logic program $P$.

Definition 7 (Satisfaction) The encapsulation of an epistemic 'object' logic program $P$ into a 'meta' program $P^{A}$ means that, for any consistent many-valued Herbrand interpretation $I \in \mathcal{B}^{H_{P}}$ and its extension $v_{B}: \mathcal{L} \rightarrow \mathcal{B}$, the function symbols $\kappa_{p}=\mathcal{K}(p), p \in P_{S}$ reflect this semantics, i.e.

for any tuple $\quad \boldsymbol{c} \in \mathcal{T}_{0}^{\text {arity }(p)}, \quad \kappa_{p}(\boldsymbol{c})=I(p(\boldsymbol{c}))$.

So, we obtain a mapping, $\quad \Theta: \mathcal{B}^{H_{P}} \rightarrow 2^{H_{P}^{A}}$, such that $I^{A}=\Theta(I) \in 2^{H_{P}^{A}}$, with: for any ground atom $p(\boldsymbol{c})$, $I^{A}(\mathcal{E}(p(\boldsymbol{c})))=t$, if $\kappa_{p}(\boldsymbol{c})=I(p(\boldsymbol{c})) ; f$ otherwise.

Let $g$ be a variable assignment which assigns values from $\mathcal{T}_{0}$ to object variables. We extend it to atoms with variables, so that $g\left(\mathcal{E}\left(p\left(x_{1}, . ., x_{n}\right)\right)\right)=\mathcal{E}\left(p\left(g\left(x_{1}\right), . ., g\left(x_{n}\right)\right)\right)$, and to all formulas in the usual way: $\psi / g$ denotes a ground formula obtained from $\psi$ by assignment $g$, then

1. $I^{A} \vDash_{g} \mathcal{E}\left(p\left(x_{1}, . ., x_{n}\right)\right) \quad$ iff $\quad \kappa_{p}\left(\left(g\left(x_{1}\right), . ., g\left(x_{n}\right)\right)\right)=$ $I\left(p\left(g\left(x_{1}\right), . ., g\left(x_{n}\right)\right)\right)$.

$I^{A} \vDash_{g} \mathcal{E}\left(\sim p\left(x_{1}, \ldots, x_{n}\right)\right) \quad$ iff $\quad \sim \kappa_{p}\left(\left(g\left(x_{1}\right), . ., g\left(x_{n}\right)\right)\right)=$ $I\left(p\left(g\left(x_{1}\right), . ., g\left(x_{n}\right)\right)\right)$.

2. $I^{A} \vDash_{g} \mathcal{E}(\phi \wedge \psi) \quad$ iff $\quad I^{A} \vDash_{g} \mathcal{E}(\phi)$ and $I^{A} \vDash_{g} \mathcal{E}(\psi)$. 3. $I^{A} \vDash_{g} \mathcal{E}(\phi \vee \psi) \quad$ iff $\quad I^{A} \vDash_{g} \mathcal{E}(\phi)$ or $I^{A} \vDash_{g} \mathcal{E}(\psi)$. 4. $I^{A} \models_{g} \mathcal{E}(\phi \leftarrow \psi) \quad$ iff $\quad v_{B}(\phi / g \leftarrow \psi / g)=t$.

Notice that in this semantics the 'meta' implication $\leftarrow^{A}$, in $\mathcal{E}(\phi) \leftarrow{ }^{A} \mathcal{E}(\psi)=\mathcal{E}(\phi \leftarrow \psi)$, is based on the intuitionistic many-valued implication $\leftarrow$ (which is not classical, i.e., $\phi \leftarrow$ $\psi \neq \phi \vee \sim \psi$ ) and determines how the logical value of a body of clause "propagates" to its head.

Remark: The encapsulated logic program, obtained by the transformation of a many-valued logic program, is not the programming language for building logic programs.

It is very different from the Annotated Logic Programming (ALP) [19]: the truth of the encapsulated ground atom $p(\mathbf{c}, \alpha)$, obtained from some atom $p(\mathbf{c})$ of the original many-valued logic program, is relative to the particular many-valued interpretation $I: H_{P} \rightarrow \mathcal{B}$, that is, it is true iff $I(p(\mathbf{c}))=\alpha$, while in ALP, the annotated atom $p(\mathbf{c}): \alpha$ is true for any interpretation for which $I(p(\mathbf{c})) \leq_{k} \alpha$.

Theorem 1 The semantics of encapsulation $\mathcal{E}$ is obtained by identifying the semantic-reflection with the $\lambda$-abstraction of Generalized Herbrand interpretation, $\mathcal{K}=\lambda \mathcal{I}$, so that the semantics of many-valued logic programs can be determined by $\mathcal{I}$ (at 'object' level) or, equivalently, by its reflection $\mathcal{K}$ (at encapsulated or 'meta' level).
Proof: From $\mathcal{K}=\lambda \mathcal{I}$ we obtain that for any $p(\mathbf{c}) \in H_{P}$ holds $I(p(\mathbf{c}))=\mathcal{I}(p, \mathbf{c})=\lambda \mathcal{I}(p)(\mathbf{c})=\mathcal{K}(p)(\mathbf{c})=\kappa_{p}(\mathbf{c})$, what is the semantic of encapsulation.

We can consider the $\lambda$-abstraction of Generalized Herbrand interpretation as an epistemic semantics, because, given a Herbrand (epistemic) interpretation $I: H_{P} \rightarrow \mathcal{B}$, then for any predicate symbol $p$ and constant $\mathbf{c} \in \mathcal{T}_{0}^{\operatorname{arity}(p)}$, holds $\lambda \mathcal{I}(p)(\mathbf{c})=I(p(\mathbf{c}))$. Then the semantic of encapsulation may be defined as follows:

" ontological semantic-reflection $\equiv$ epistemic semantics", that is, $\mathcal{K}=\lambda \mathcal{I}$.

Notice that at 'meta' (ontological) level( differently from $\wedge, \vee$, which are classic 2-value boolean operators), the semantics for 'meta' implication operator, $I^{A} \vDash_{g} \mathcal{E}(\phi \leftarrow \psi)$, is not defined on $I^{A} \vDash_{g} \mathcal{E}(\phi)$ and $I^{A} \vDash_{g} \mathcal{E}(\psi)$. For example, let $I^{A} \vDash_{g} \mathcal{E}(p(\mathbf{c}))$ and $I^{A} \vDash_{g} \mathcal{E}(q(\mathbf{d}))$, with $\kappa_{p}(\mathbf{c})=f$ and $\kappa_{q}(\mathbf{d})=t$ : then $p(\mathbf{c}) \leftarrow q(\mathbf{d})$ is false and, consequently, does not hold $I^{A} \vDash \mathcal{E}(p(\mathbf{c}) \leftarrow q(\mathbf{d}))$.

Proposition $2 \quad I^{A} \vDash_{g} \mathcal{E}(\phi) \leftarrow{ }^{A} \mathcal{E}(\psi)$ implies $I^{A} \vDash_{g} \mathcal{E}(\phi)$ and $I^{A} \vDash_{g} \mathcal{E}(\psi)$, but not viceversa. The truth of $\mathcal{E}(\phi / g)$ and $\mathcal{E}(\psi / g)$ are necessary but not sufficient conditions for the truth of $\mathcal{E}(\phi / g) \leftarrow^{A} \mathcal{E}(\psi / g)$.

Following the standard definitions, we say that an interpretation $I^{A}$, of a program $P^{A}$, is a model of a $P^{A}$ if and only if every clause of $P^{A}$ is satisfied in $I^{A}$. In this way we define a model theoretic semantics for encapsulated logic programs. A set of formulas $S$, of encapsulated logic EMV, logically entails a formula $\phi$, denoted $S \vDash \phi$, if and only if every model of $S$ is also a model of $\phi$.

\section{MANY-VALUED INFERENCE CLOSURE}

Let us consider the bilattice structure $\left(\mathcal{B}_{W}, \wedge, \vee, \bullet,+, \sim\right)$ of the Definition 2, where $\mathcal{B}_{W}$ denotes the set $\mathcal{P}(W) \times \mathcal{P}(W)$, and a collection of world corresponds to the set of Herbrand interpretations over a given Herbrand base $H_{P}$, i.e., $W=$ $\mathcal{B}^{H_{P}}$. Now, with this new setting, we review the general framework proposed by Ginsberg [11].

Let us consider a world-bilattice $\mathcal{B}_{W}$ a set of truth values for set of formulae in $\mathcal{L}^{A}$. Recall that a truth assignment for the bilattice $\mathcal{B}_{W}$, in this case, is a mapping: $\Phi: \mathcal{L}^{A} \rightarrow \mathcal{B}_{W}$. so that for any $\mathcal{E}(\psi) \in \mathcal{L}^{A}$, where $\psi \in \mathcal{L}$, the $(U, V)=$ $\Phi(\mathcal{E}(\psi))$ is a pair of subsets of $W$ : the subset $U$ is the set of worlds (Herbrand interpretations) where $\mathcal{E}(\psi)$ is true, while the subset $V$ is the set of worlds (Herbrand interpretations) where $\mathcal{E}(\psi)$ is false.

We can project this mapping onto the first component of $\mathcal{B}_{W}=\mathcal{P}(W) \times \mathcal{P}(W)$ to set another truth assignment $\Phi_{+}: \mathcal{L}^{A} \rightarrow \mathcal{P}(W)$.

$\Phi_{+}$takes a sentence $\mathcal{E}(\psi) \in \mathcal{L}^{A}$ and maps it to the collection of worlds where it is known to hold. As a function from $\mathcal{L}^{A}$ to $\mathcal{P}(W), \Phi_{+}$is nothing more than a relation on $\mathcal{L}^{A}$ and $W$, with $\Phi_{+}(\mathcal{E}(\psi), w)$ if and only if $\mathcal{E}(\psi)$ is given as true in the world $w$.

We can easily interpret this as a function

$\widetilde{\Phi}_{+}: W \rightarrow \mathcal{P}\left(\mathcal{L}^{A}\right)$.

which, given a world $w$ (i.e. a Herbrand interpretation), 
produces the set of 'meta' (encapsulated) sentences known to be true in it.

Now we are ready to define the world-closed ( $w$-closed) manyvalued truth assignment (valuation) $v_{B}: \mathcal{L} \rightarrow \mathcal{B}$ given in the Definition 4.

Definition 8 Let $v_{B}: \mathcal{L} \rightarrow \mathcal{B}$ be a truth assignment, obtained as extended valuation of a Herbrand interpretation $I=w$ : $H_{P} \rightarrow \mathcal{B}$ (Def. 5), then we define the 'meta' valuation $v_{B}^{A}:$ $\mathcal{L}^{A} \rightarrow 2$ as follows: for any $p(\boldsymbol{c}) \in H_{P}$ and $\psi, \varphi \in \mathcal{L}$,

1. $v_{B}^{A}\left(\mathcal{E}(p(\boldsymbol{c}))=\mathcal{E}\left(v_{B}(p(\boldsymbol{c}))=\kappa_{p}(\boldsymbol{c})\right)\right.$;

$v_{B}^{A}\left(\mathcal{E}(\sim p(\boldsymbol{c}))=\mathcal{E}\left(v_{B}(p(\boldsymbol{c}))=\sim \kappa_{p}(\boldsymbol{c})\right)\right.$;

2. $v_{B}^{A}(\mathcal{E}(\psi) \odot \mathcal{E}(\varphi))=v_{B}^{A}(\mathcal{E}(\psi)) \odot v_{B}^{A}(\mathcal{E}(\varphi))$;

where $\odot \in\{\wedge, \vee\}$;

3. $v_{B}^{A}\left(\mathcal{E}(\psi) \leftarrow{ }^{A} \mathcal{E}(\varphi)\right)=\mathcal{E}\left(v_{B}(\psi \leftarrow \varphi)\right)$;

Let $\mathcal{L}_{W}={ }_{\text {def }}\left\{\phi=\mathcal{E}(\psi) \mid \psi \in \mathcal{L}\right.$ and $\left.v_{B}^{A}(\phi)=t\right\}$, then a many-valued Herbrand interpretation $I: H_{P} \rightarrow \mathcal{B}$ is w-closed iff

(a) $\widetilde{\Phi}_{+}(w)$ is deductively closed (as subset of $\mathcal{L}^{A}$ ) for each $w \in W$, and

(b) $I=\bigcap_{\phi \in \mathcal{L}_{W}} \Phi_{+}(\phi)$, with $\phi \notin \mathcal{L}_{W}$ implies $I \notin \Phi_{+}(\phi)$. The extended many-valued valuation (truth assignment) $v_{B}$ : $\mathcal{L} \rightarrow \mathcal{B}$ of the $w$-closed Herbrand interpretation $I: H_{P} \rightarrow \mathcal{B}$, is $w$-closed also.

The approach we are following for this 'meta' (encapsulated) 2 -valued logic is quite close to that used to define the closure operation in first-order (2-valued) logic itself: the fixed set $\widetilde{\Phi}_{+}(w)$ is deductively closed (that $\phi \wedge \sigma$ be in the set if $\phi$ and $\sigma$ are, for example) if it is the intersection of all of its closed supersets. This definition makes sense because the intersection of closed sets is itself closed. For many-valued truth assignment closure holds this analog fact:

Proposition 3 Let $v_{B}, u_{B} \in \mathcal{B}^{\mathcal{L}}$ be two $w$-closed manyvalued truth assignments. Then $v_{B} \otimes u_{B}$ is also $w$-closed. In general, if $v_{B_{k}}$ are all $w$-closed, than so is

$\prod_{k} v_{B_{k}}=v_{B_{1}} \otimes \ldots \otimes v_{B_{n}}$.

where, if we represent truth assignments by triples $[T, P, F]$, (of true, possible, and false subsets of $\mathcal{L}$ respectively; the remaining part of $\mathcal{L}$ is considered as unknown), then the meet knowledge operation $\otimes$ is defined by

$[T, P, F] \otimes\left[T_{1}, P_{1}, F_{1}\right]=\left[T \bigcap T_{1}, P \bigcap P_{1}, F \bigcap F_{1}\right]$.

So we can mimic the usual (set-based) definition of closure:

Definition 9 The $w$-closure of many-valued truth assignment $v_{B}$ is given by

$\operatorname{cl}\left(v_{B}\right)=\prod\left\{u_{B} \mid u_{B} \geq_{k} v_{B} \quad\right.$ and $u_{B}$ is w-closed $\}$

In his paper [11] Ginsberg tried to define inference-based closure operation $c l$ for truth assignments $v_{B}: \mathcal{L} \rightarrow \mathcal{B}$, based on the following four principles:

1. It should be a bilattice construction, depending only on the partial orders and operation of bilattice. Here we will consider only Belnap's 4-valued distributive bilattice.

2. It should accurately reflect the notion of inference already formalized in existing work on extensions to first-order logic.
3. $\operatorname{cl}\left(\operatorname{cl}\left(v_{B}\right)\right)=\operatorname{cl}\left(v_{B}\right)$ for all truth assignments $v_{B}$.

4. $\operatorname{cl}\left(v_{B}\right)(\psi) \geq_{k} \quad v_{B}(\psi)$ for all $\psi \in \mathcal{L}$.

where the last two principles are general requirements for any closure operation.

It is easy to verify that one of the conditions on a truth assignment $v_{B}$ being w-closed is that $\widetilde{\Phi}_{+}$be deductively closed. This notion, however, is not bilattice-theoretic. Thus in [11] Ginsberg tried to give an equivalent definition of closure that does not suffer from this deficiency, by the following definition of the apparent closure (a-closure):

Definition 10 [11] A truth assignment $v_{B}: \mathcal{L} \rightarrow \mathcal{B}$ will be called a-closed if:

1. if $\psi \vDash_{\mathcal{B}} \phi$, then $v_{B}(\phi) \geq_{t} v_{B}(\psi)$,

2. $v_{B}\left(\wedge_{i} \psi_{i}\right) \geq_{k} \wedge_{i} v_{B}\left(\psi_{i}\right)$, and

3. $v_{B}(\sim \psi)=\sim v_{B}(\psi)$ for all $\psi$.

But, as critically pointed Schaerf in [20], these conditions (particularly first one) show very strong assumptions about proposed logic. What we are really imposing here is a classical (2-valued) semantics to a many-valued setting: this framework collapses in the presence of contradictions. If we have a proposition which assumes the top knowledge truth value $T$, then all the propositions will assume the truth value T. As consequence, in the 4-valued Belnap's logic we can use only three logic values. "For this reason, the search for alternative foundation for Ginsberg's framework is still wide open and, in our opinion, crucial for future development" concluded Shaerf in [20].

What follows is just the positive answer to this open problem, and is valid for any (not only 4-valued Belnap's logic) manyvalued logic.

The notion of logic entailment (or model-theoretic consequence relation) and of logic validity in a 2 -valued logic, given by the syntax $p \vDash q$, (" $q$ is a consequence of $p ")$, holds when the set of all models where $p$ is true is contained in the set of all models where $q$ is true. The difficulty for many-valued logics is in the understanding of how to define logic entailment when, for instance, the falsity of $q$ is a consequence of the possibility of $p$. More over, let $\triangle$ be the many-valued set of formulae in which each formula has some fixed value (not obligatorily the true value) in $\mathcal{B}$, so that we can conclude that $q$ has a possible value: such conclusion is valid if the set of all models where the formulae in $\triangle$ has these fixed values, is contained in the set of models where $q$ has a possible value. Such, more complex relation, can be given by the classic 2-valued logic entailment, not for original ('object') many-valued formulae, but for their encapsulated 'meta' formulae, i.e., by the classic 2-valued consequence relation $\mathcal{E}(\triangle) \vDash \mathcal{E}(q)$. Thus we are able to substitute the 2-valued (at 'object' level) consequence relation used by Ginsberg in order to define a-closure, with this 'encapsulated' 2-valued consequence relation:

Definition 11 A truth assignment $v_{B}: \mathcal{L} \rightarrow \mathcal{B}$ will be called a-closed if:

if $\mathcal{E}(\psi) \vDash \mathcal{E}(\phi)$, then $v_{B}^{A}(\mathcal{E}(\phi)) \geq_{t} v_{B}^{A}(\mathcal{E}(\psi))$, where $\psi, \phi \in \mathcal{L}$ and $v_{B}^{A}: \mathcal{L}^{A} \rightarrow 2$ is the 'meta'valuation determined from $v_{B}$, and, $\quad \psi \vDash_{\mathcal{B}} \phi \quad$ iff $\mathcal{E}(\psi) \vDash \mathcal{E}(\phi)$. 
Notice that the second condition in Ginsberg's Definition 10 is replaced by the more general (see Belnap's and Fitting's considerations) condition 3 of Definition 4; while the third condition is common for all valuations (condition 2 of Definition 4).

Example for $\vDash_{\mathcal{B}}$ : Let $\triangle=\left\{p_{1}\left(\mathbf{c}_{1}\right), \ldots, p_{n}\left(\mathbf{c}_{n}\right)\right\}$ be a set of ground atoms, with values $\alpha_{1}, \ldots, \alpha_{n}$ respectively, of a many-valued logic program, so that $\mathcal{E}(\triangle)=\mathcal{E}\left(p_{1}\left(\mathbf{c}_{1}\right)\right) \wedge \ldots \wedge$ $\mathcal{E}\left(p_{n}\left(\mathbf{c}_{n}\right)\right)=p_{1}^{A}\left(\mathbf{c}_{1}, \alpha_{1}\right) \wedge \ldots \wedge p_{n}^{A}\left(\mathbf{c}_{n}, \alpha_{n}\right)$, and $\mathcal{E}(p(\mathbf{c}))=$ $p^{A}(\mathbf{c}, \alpha)$, then $\mathcal{E}(\triangle) \vDash \mathcal{E}(p(\mathbf{c}))$, that is, $\triangle \vDash_{\mathcal{B}} p(\mathbf{c})$, means that the ground atom $p(\mathbf{c})$ with this value $\alpha$ is (many-valued) inferred from the set of ground atoms $\triangle$, if the set of all manyvalued models where $p(\mathbf{c})$ has a value $\alpha \in \mathcal{B}$ contains the set of many-valued models where for each $1 \leq i \leq n, p_{i}\left(\mathbf{c}_{i}\right)$ has the value $\alpha_{i}$.

Thus, we are able to define the many-valued, bilattice-theoretic dependent, inference closure:

Theorem 2 A many-valued truth assignment is apparently closed if and only if it is $w$-closed. Thus,

$\operatorname{cl}\left(v_{B}\right)=\prod\left\{u_{B} \mid u_{B} \geq_{k} v_{B} \quad\right.$ and $u_{B}$ is a-closed $\}$. cl is monotonic, that is, if $\phi \leq_{k} \varphi$, then $\operatorname{cl}(\phi) \leq_{k} \operatorname{cl}(\varphi)$.

Proof: 1. From w-closed to a-closed: Let $v_{B}: \mathcal{L} \rightarrow \mathcal{B}$ be w-closed. If $\mathcal{E}(\psi) \vDash \mathcal{E}(\phi)$, then $\mathcal{E}(\phi)$ will be true in at least as many worlds as $\mathcal{E}(\psi)$ is, and can be false in no more. Thus $v_{B}^{A}(\mathcal{E}(\phi)) \geq_{t} v_{B}^{A}(\mathcal{E}(\psi))$, i.e., $v_{B}: \mathcal{L} \rightarrow \mathcal{B}$ is a-closed.

2. From a-closed to w-closed: Suppose that $v_{B}: \mathcal{L} \rightarrow \mathcal{B}$ is a-closed, but not w-closed. Thus, must be some $w \in W$ such that $\widetilde{\Phi}_{+}(w)$ is not deductively closed. There must therefore be some collection $\mathcal{E}\left(\psi_{1}\right), \ldots, \mathcal{E}\left(\psi_{n}\right)$ with $\mathcal{E}\left(\psi_{i}\right) \in \widetilde{\Phi}_{+}(w)$ and $\mathcal{E}\left(\psi_{1}\right), \ldots, \mathcal{E}\left(\psi_{n}\right) \vDash \mathcal{E}(\phi)$ but $\mathcal{E}(\phi) \notin \widetilde{\Phi}_{+}(w)$. But since $v_{B}$ is a-closed,

$\wedge_{i} v_{B}^{A}\left(\mathcal{E}\left(\psi_{i}\right)\right)=v_{B}^{A}\left(\wedge_{i} \mathcal{E}\left(\psi_{i}\right)\right)=v_{B}^{A}\left(\mathcal{E}\left(\wedge_{i} \psi_{i}\right)\right) \leq_{t} v_{B}^{A}(\mathcal{E}(\phi))$ It follows that (from $\mathcal{E}\left(\psi_{i}\right) \in \widetilde{\Phi}_{+}(w)$ )

$w \quad \in \quad \bigcap_{i} \Phi_{+}\left(\mathcal{E}\left(\psi_{i}\right)\right) \quad \subseteq \quad \Phi_{+}\left(\wedge_{i} \mathcal{E}(\psi)\right) \quad=$ $\Phi_{+}\left(\mathcal{E}\left(\wedge_{i} \psi\right)\right) \subseteq \Phi_{+}(\mathcal{E}(\phi))$

or $w \in \Phi_{+}(\mathcal{E}(\phi))$, so that $\left.\mathcal{E}(\phi)\right) \in \widetilde{\Phi}_{+}(w)$ after all. This contradiction completes the proof. The monotonicity of $\mathrm{cl}$ follows immediately from the fact that the set over which the product is taken varies k-monotonically with the truth assignment in question.

\section{CONCLUSiON}

We have presented a many-valued programming logic with the intuitionistic 4-valued implication, capable of handling incomplete and inconsistent beliefs, based on the revisited 4valued Belnap's bilattice, by introducing the possible logic value instead of inconsistent (both true and false), which has clear model theory and fixed point semantics. In the process of the encapsulation we distinguish two levels: the epistemic many-valued level of ordinary logic programs with negation based on a bilattice operators, and the ontological 'meta' level of encapsulated logic programs. Such encapsulation is a semantic-reflection at ontological level of the epistemic semantics at many-valued object level, and is a homomorphism between these two logic levels. In this way we are able to formalize the logical entailment for a many-valued logic, by considering the main criticism to Ginsberg's approach given by Schaerf, consisting in the lack of a multivalued semantics and the use of the notion of validity in 2-valued case even when dealing with more complex sets of truth values. The resulting ontological 2-valued logic, which encapsulates the semantics of many-valued logics is not an issue "per se": it is considered as a minimal logic tool (differently from more complex logics, as annotated and signed logics) useful to define many-valued entailment and inference. But, recently, it is used also in order to define the coalgebraic semantics for logic programs [16], and in the future, considering the fact that for any predicate at many-valued level, by encapsulation we obtain a new predicate, with one logic-attribute extension (for the epistemic logic value), we will consider the possibility to use the ordinary relational database schemas with such extended predicates in global schemas of data integration systems, for query answering from their many-valued Herbrand model.

\section{REFERENCES}

[1] M.C.Fitting, "A Kripke/Kleene semantics for logic programs," Journal of Logic Programming 2, pp. 295-312, 1985.

[2] K.Kunen, "Negation in logic programming," Journal of Logic Programming 4, pp. 289-308, 1987

[3] T.Przymusinski, "Every logic program has a natural stratification and an iterated fixed point model," In Eighth ACM Symposium on Principles of Databases Systems, pp. 11-21, 1989.

[4] T.Przymusinski, "Well-founded semantics coincides with thre-valued stable-semantics," Fundamenta Informaticae 13, pp. 445-463, 1990.

[5] J.W.Lloyd, Foundations of LOgic Programming, Springer-Verlag, New York, 1984

[6] N.D.Belnap, "A useful four-valued logic," In J-M.Dunn and G.Epstein, editors, Modern Uses of Multiple-Valued Logic. D.Reidel, 1977.

[7] M.C.Fitting, "Billatices and the semantics of logic programming," Journal of Logic Programming, 11, pp. 91-116, 1991.

[8] M.C.Fitting, "Billatices are nice things," Proceedings of Conference on Self-Reference, Copenhagen, 2002.

[9] M.Ginsberg, "Multivalued logics: A uniform approach to reasoning in artificial intelligence," Computational Intelligence, vol.4, pp. 265-316, 1988.

[10] Z. Majkić, "Meta many-valued logic programming for incomplete and locally inconsistent databases," 8th International Database Engineering and Application Symposium (IDEAS),July 7-9, Coimbra, Portugal, 2004.

[11] M.L.Ginsberg, "Bilattices and modal operators," Tech.Rep.N.94305, Comp.Science Dept. Stanford University, California, 1990.

[12] Robert C. Moore, "Semantical considerations on nonmonotonic logic," Acta Informatica, vol. 25, pp. 75-94, 1985.

[13] Z. Majkić, "Autoepistemic bilattice-based logic programming for reasoning with inconsistency," Notes in http://www.dis.uniromal.it/ majkic/, 2004.

[14] Z. Majkić, "Ontological encapsulation of many-valued logic," 19th Italian Symposium of Computational Logic (CILC04),June 16-17, Parma, Italy, 2004.

[15] Z. Majkić, "Truth and knowlwdge fixpoint semantics for many-valued logic programming," 19th Workshop on (Constraint) Logic Programming (W(C)LP 2005), February 21-25, Ulm, Germany, 2005.

[16] Z. Majkić, "Coalgebraic semantics for logic programming," 18th Worshop on (Constraint) Logic Programming, W(C)LP 2004, March 0406, Berlin, Germany, 2004.

[17] F.Bry, "Logic programming as constructivism: a formalization and its application to databases," In Proc. of the Symposium on Principles of Database Systems, ACM SIGACT-SIGMOD, 1989.

[18] H.Rasiowa and R.Sikorski, "The matematics of metamatematics," $P W N$ Polisch Scientific Publishers, Warsaw, 3rd edition, 1970.

[19] M.Kifer and V.S.Subrahmanian, "Theory of generalized annotated logic programming and its applications," Journal of Logic Programming 12(4), pp. 335-368, 1992.

[20] M.Schaerf, "Notes on Ginsberg's multivalued logics," Comput. Intell., vol. 7, pp. 154-159, 1991. 